\title{
Self-reported adherence to oral cancer therapy: relationships with symptom distress, depression, and personal characteristics
}

This article was published in the following Dove Press journal:

Patient Preference and Adherence

4 November 2015

Number of times this article has been viewed

\section{Donna L Berry ${ }^{1-3}$ \\ Traci M Blonquist ${ }^{4}$ \\ Fangxin Hong ${ }^{4,5}$ \\ Barbara Halpenny' \\ Ann H Partridge ${ }^{2,3}$}

'Phyllis F Cantor Center, Dana-Farber Cancer Institute, ${ }^{2}$ Medical Oncology, Department of Medicine, Dana-Farber Cancer Institute, ${ }^{3}$ Department of Medicine, Harvard Medical School, ${ }^{4}$ Department of Biostatistics and Computational Biology, Dana-Farber Cancer Institute, ${ }^{5}$ Department of Biostatistics, Harvard School of Public Health, Boston, MA, USA
Correspondence: Donna L Berry Phyllis F Cantor Center, Dana-Farber Cancer Institute, 450 Brookline Avenue, LW-518, Boston, MA 02115, USA

Tel + I 6176321909

Fax + I 6175828550

Email donna_berry@dfci.harvard.edu
Background: Therapeutic cancer chemotherapy is most successful when complete dosing is achieved. Because many newer therapeutic agents are oral and self-administered by the patient, adherence is a concern. The purpose of our analysis was to explore relationships between adherence, patient characteristics, and barriers to adherence.

Methods: This secondary analysis utilized self-reported data from a randomized trial of self-care management conducted at two cancer centers in the US. Symptom distress was measured using the 15-item Symptom Distress Scale (SDS-15) and depression with the Patient Health Questionnaire-9 (PHQ-9). Adherence to oral medication was self-reported using the 8-item Morisky Medication Adherence Scale (MMAS-8). Measures were collected via Web-based, study-specific software $\sim 8$ weeks after treatment start date. Odds of low/medium adherence (score $<8$ ) were explored using univariate logistic regression. Given the number of factors and possible relationships among factors, a classification tree was built in lieu of a multivariable logistic regression model.

Results: Of the eligible participants enrolled, 77 were on oral therapy and 70 had an MMAS score. Forty-nine $(70 \%)$ reported a high adherence score $(=8)$. Higher odds of low/medium adherence were associated with greater symptom distress $(P=0.09)$, more depression $(P=0.05)$, chemotherapy vs hormonal oral medication $(P=0.03)$, being female $(P=0.02)$, and being randomized to the control group in the parent trial $(P=0.09)$. Conversely, high adherence was associated with working ( $P=0.08)$, being married/partnered $(P=0.004)$, and being older $(P=0.02)$. Factors identified as significantly related to low/medium adherence from the univariate logistic regression analyses were supported by the classification tree results.

Conclusion: Nonadherence to therapeutic oral medications in patients with cancer was associated with being unmarried/unpartnered, symptom distress, younger age, not working, and female sex. These findings may help to identify patients at risk for nonadherence and for whom supportive interventions to enhance adherence may be needed.

Keywords: patient-centered technology, self-management, logistic regression, recursive partitioning

\section{Introduction}

Much attention has been focused on patient adherence to oral chemotherapy, primarily prompted by the increasing clinical use of approved and experimental oral targeted therapies. Successful chemotherapy is best achieved by complete dosing of the prescribed agent whether it be an intravenous infusion in the clinic or a patientadministered oral medication. ${ }^{1}$ The Clinical Journal of Oncology Nursing dedicated an entire issue to the topic in June 2015, and the American Society of Clinical Oncology and the Oncology Nursing Society published comprehensive guidelines covering the safety and administration of oral chemotherapy in $2013 .{ }^{2}$ Furthermore, 
various other types of therapeutic oral medications (eg, antiestrogens, antiandrogens) or those intended to prevent severe toxicities (eg, allopurinol) are prescribed to patients with cancer. Clinical investigators have studied adherence since the $1980 \mathrm{~s}^{3}$ with varying results. Authors of systematic reviews ${ }^{4-6}$ have identified factors that interfere with, or promote, patient adherence to oral medications. Factors relevant to the characteristics of the patient, the regimen, and its side effects, as well as the institutional and home environments, have been implicated. Johnson ${ }^{4}$ listed factors that promoted adherence with large effect sizes when studied, identifying positive provider relationships, low side-effect profiles, high knowledge levels about the medications, and family support. Mathes et $\mathrm{al}^{5}$ discussed the fact that oral agent side effects are not always strong predictors of low adherence. A number of programs of research have focused on developing interventions to improve or ensure adherence to oral medications. ${ }^{7,8}$ More recently, Spoelstra and Sansoucie ${ }^{9}$ classified interventions that were "recommended for practice" based on strong evidence for promoting adherence that included patient monitoring, feedback, and interventions combining patient education and support with various methods of reminders, packaging, and feedback.

While conducting a randomized trial ${ }^{10}$ of a Web-based, patient-centered, educational intervention during active cancer therapy in which symptom distress was a primary outcome, we took the opportunity to assess adherence to oral medications. The trial was approved by the Dana-Farber/Harvard Cancer Center Institutional Review Board. The purpose of this analysis was to explore oral agent adherence in relationship to the study group, cancer symptoms, type of agent, psychosocial measures, and selected demographic variables.

\section{Methods}

\section{Sample and procedures}

This secondary analysis utilized self-reported data from the randomized Electronic Self-Report Assessment for Cancer (ESRA-CII) trial conducted at two comprehensive cancer centers. The details of the trial have been reported elsewhere. ${ }^{10}$ To summarize, a total of 779 adult, ambulatory patients with cancer of any type who had started a new therapeutic regimen were enrolled and randomized; 752 were deemed eligible. All participants used the Web-based ESRA-C to self-report symptoms and quality of life prior to starting a new cancer therapy (T1), at 3-6 weeks (T2), 6-8 weeks after T2 (T3), and at the end of the therapeutic regimen (T4). The intervention group participants were presented with teaching tips for symptoms and quality of life issues (SxQOL), which were reported above a predetermined threshold. The education included why and how often a particular SxQOL happens, what to do at home for selfcare, and when to call the clinic. Monitoring and tracking of SxQOL was available to the intervention group as well, within the ESRA-C program.

\section{Measures}

Symptom distress was measured using the 15-item Symptom Distress Scale (SDS-15) ${ }^{10,11}$ and depression with the Patient Health Questionnaire (PHQ-9). ${ }^{12,13}$ Participants who were prescribed any type of therapeutic oral agent were identified by medical record review. Adherence to oral medication was collected at T3 using the 8-item Morisky Medication Adherence Scale (MMAS-8) ${ }^{14}$ adapted to the cancer setting. ${ }^{15}$ Two patients were given the MMAS- 8 at T4 and the corresponding symptom measures were used. An additional adherence item was asked, with the permission of the MMAS-8 creators, to evaluate overadherence.

\section{Analytic procedure}

The T3 data for adherence, symptom distress, and depression were analyzed among participants on oral therapy. The frequency with which participants indicated a nonadherent behavior was summarized for each item in the MMAS- 8 scale and the additional item. The overadherence item was not used in the computation of the overall adherence score. The MMAS- 8 score was categorized into low/medium (score $<8$ ) and high (score $=8$ ) adherence. ${ }^{14}$ The odds of low/medium adherence were explored univariately using logistic regression. On the basis of previous research, our factors of interest included the following: SDS-15 summed score, PHQ-9 summed score, months on oral medication, therapy type (chemotherapy vs hormonal), sex, stage (IV vs 0-III), study group, work status, education ( $\leq$ high school vs $>$ high school), minority status, relationship status (married/partnered vs not), and age.

Given the small sample size relative to the number of factors and possible relationships among factors, a classification tree subsequently was built in lieu of a multivariable logistic regression model. Classification trees comprise a nonparametric approach that performs well when there is a complex relationship between the factors and outcome. ${ }^{16}$ Recursive partitioning was implemented to build the classification tree exploring the most influential factors related to low/ medium adherence using the Rpart functionality in $\mathrm{R} .{ }^{17}$ The tree was built by first identifying the factor that best split the data into low/medium-adherence and high-adherence nodes. Each node had the potential to be split again if the sample 
size was adequate and the split was considered beneficial. The minimum number of participants required in a node to consider a further split was 15 participants. A terminal node was defined when no further splits occurred. Rpart uses the Gini index setting to determine if a split is beneficial. ${ }^{17}$ The default ten cross-validations were conducted with the procedure. The classification rate, or proportion of correct classifications divided by the number of observations in a node, was calculated for each terminal node. All analyses were performed in SAS Version 9.2 and R 2.15.2. Due to the exploratory nature of this analysis, a significance level of 0.1 was used to explore a relationship among study variables.

\section{Results}

Of the 752 eligible participants enrolled in the randomized trial, 77 were on oral therapy at T3, and 70 of these had an
MMAS score. Patient characteristics are provided in Table 1 for those on oral therapies with an adherence score. Among the 70 participants, $42(60 \%)$ were male, age ranged from 34 years to 80 years (median $=61$ years), and $22(31 \%)$ had stage IV disease. The most common cancer diagnoses included 31 prostate (44\%) and 19 breast (27\%). Forty-nine (70\%) reported an adherence score of 8.0, indicating high adherence. High adherence was reported in 16 of $29(55 \%)$ participants taking oral chemotherapy and 33 of 41 (80\%) taking hormonal medications. The most frequent reason why a participant did not adhere was forgetting to take the oral medication (Table 2). Only one participant reported taking more than the prescribed dose.

The results from the univariate analyses (Table 3 ) suggest that higher odds of low/medium adherence were associated with higher symptom distress $(P=0.09)$, higher depression

Table I Patient characteristics for all $(\mathrm{N}=70)$ and for those with self-reported low/medium adherence and high adherence to oral cancer medications

\begin{tabular}{|c|c|c|c|}
\hline \multirow[t]{2}{*}{ Patient characteristic } & \multirow[t]{2}{*}{ Overall N (\%) } & \multirow{2}{*}{$\begin{array}{l}\text { Low/medium adherence } \\
\mathbf{N}(\%)\end{array}$} & \multirow{2}{*}{$\begin{array}{l}\text { High adherence } \\
\mathbf{N}(\%)\end{array}$} \\
\hline & & & \\
\hline $\mathrm{N}$ & 70 & 21 & 49 \\
\hline Age, median (range), years & $61(34-80)$ & $56(34-75)$ & $62(39-80)$ \\
\hline Minority & $9(14)$ & $3(14)$ & $6(12)$ \\
\hline \multicolumn{4}{|l|}{ Sex } \\
\hline Male & $42(60)$ & $8(38)$ & $34(69)$ \\
\hline Female & $28(40)$ & $13(62)$ & $15(31)$ \\
\hline \multicolumn{4}{|l|}{ Study group } \\
\hline Control & $39(56)$ & $15(7 \mid)$ & $24(49)$ \\
\hline Intervention & $31(44)$ & $6(29)$ & $25(5 \mathrm{I})$ \\
\hline \multicolumn{4}{|l|}{ Cancer type } \\
\hline Breast & $19(27)$ & $6(29)$ & $13(27)$ \\
\hline Colorectal & $\mathrm{I}(\mathrm{I})$ & I (5) & $0(0)$ \\
\hline Other GI & $6(9)$ & $2(10)$ & $4(8)$ \\
\hline Prostate & $31(44)$ & $6(29)$ & $25(5 \mathrm{I})$ \\
\hline Renal cell & $6(9)$ & $3(14)$ & $3(6)$ \\
\hline Sarcoma & $5(7)$ & $2(10)$ & $3(6)$ \\
\hline Other & $2(3)$ & I (5) & $I(2)$ \\
\hline \multicolumn{4}{|l|}{ Therapy type } \\
\hline Chemotherapy & $29(4 I)$ & $13(62)$ & $16(33)$ \\
\hline Hormonal therapy & $41(59)$ & $8(38)$ & $33(67)$ \\
\hline Months on oral agents, median (range) & $2.27(0.39-14.01)$ & $2.10(0.46-14.01)$ & $2.34(0.39-4.70)$ \\
\hline \multicolumn{4}{|l|}{ Stage } \\
\hline 0 & $2(3)$ & $2(10)$ & $0(0)$ \\
\hline I & $8(I I)$ & $2(10)$ & $6(12)$ \\
\hline 2 & $27(39)$ & $3(14)$ & $24(49)$ \\
\hline 3 & $10(14)$ & $4(19)$ & $6(12)$ \\
\hline 4 & $22(3 \mathrm{I})$ & $9(43)$ & $13(27)$ \\
\hline True missing* & $I(I)$ & I (5) & $0(0)$ \\
\hline Working ( $N=62)$ & $40(65)$ & $8(47)$ & $32(7 I)$ \\
\hline \multicolumn{4}{|l|}{ Education $(\mathrm{N}=69)$} \\
\hline$\leq \mathrm{HS}$ & $12(17)$ & $4(20)$ & $8(16)$ \\
\hline$>\mathrm{HS}$ & $57(83)$ & $16(80)$ & $4 \mid(84)$ \\
\hline Married/partnered & $61(87)$ & $14(67)$ & $47(96)$ \\
\hline
\end{tabular}

Note: *indicates a non-solid tumor.

Abbreviations: GI, gastrointestinal; HS, high school. 
Table 2 Item response frequencies of nonadherent behavior on the MMAS-8 adapted to the cancer setting

\begin{tabular}{|c|c|c|c|}
\hline \multirow[t]{2}{*}{ Item } & \multirow{2}{*}{$\frac{\text { Total }}{\text { N (\%) }}$} & \multirow{2}{*}{$\frac{\text { Control }}{\mathbf{N}(\%)}$} & \multirow{2}{*}{$\begin{array}{l}\text { Intervention } \\
\mathbf{N}(\%)\end{array}$} \\
\hline & & & \\
\hline & 70 & 39 & 31 \\
\hline Sometimes forget to take OCM? & $9(13)$ & $8(21)$ & I (3) \\
\hline Over past 2 weeks, any days OCM not taken? & $7(10)$ & $3(8)$ & $4(13)$ \\
\hline Ever cut back or stopped OCM without telling the doctor because you felt worse when taking it? & $I(1)$ & $0(0)$ & $\mathrm{I}(3)$ \\
\hline When traveling, do you forget to bring OCM? & $2(3)$ & $2(5)$ & $0(0)$ \\
\hline Did you take your OCM yesterday? & $7(10)$ & $4(10)$ & $3(10)$ \\
\hline When you feel cancer is under control, do you stop taking OCM? & $I(1)$ & $0(0)$ & I (3) \\
\hline Do you feel hassled about sticking to cancer plan? & $6(9)$ & $4(10)$ & $2(6)$ \\
\hline \multirow[t]{2}{*}{ How often do you have difficulty remembering to take OCM? } & I (I): sometimes & $I(I)$ & $0(0)$ \\
\hline & 5 (7): once in a while & $5(13)$ & $0(0)$ \\
\hline Have you taken more than regular dose? & $\mathrm{I}(\mathrm{I})$ & $I(3)$ & $0(0)$ \\
\hline
\end{tabular}

Note: $\mathrm{N}=70$.

Abbreviations: MMAS, Morisky Medication Adherence Scale; OCM, oral chemotherapy medicine.

$(P=0.05)$, taking chemotherapy vs hormonal oral medication ( $P=0.03)$, being female $(P=0.02)$, and being randomized to the control group $(P=0.09)$. Conversely, the odds of high adherence were associated with working $(P=0.08)$, being married/partnered $(P=0.004)$, and being older $(P=0.02)$.

The factors identified as significantly related to the odds of low/medium adherence from the univariate logistic regression analyses were supported by the classification tree results. Five splits and six terminal nodes were generated, in which nodes 1, 3, and 5 indicated low/medium adherence (Figure 1). Node 1 was defined by relationship status, specifically not married or partnered. For those who were married/partnered, Node 3 was defined by high symptom distress (SDS-15 $\geq 20$ ) and age $<46$ years. For those married/partnered and with lower symptom distress, Node 5 was defined by not working and being female. The classification rates ranged from 0.67 to 1.00 across the six terminal nodes.

Table 3 Univariate odds of low/medium adherence

\begin{tabular}{|c|c|c|}
\hline \multirow[t]{2}{*}{ Variable } & \multicolumn{2}{|l|}{ Univariate } \\
\hline & OR (95\% Cl) & $P$-value \\
\hline SDS-I5+I vs SDS-I $5^{a}$ & I.I (I.0-I.2) & 0.09 \\
\hline PHQ9C+I vs PHQ9C ${ }^{a}$ & $1.2(1.0-1.4)$ & 0.05 \\
\hline Chemotherapy vs hormonal therapy & $3.4(1.2-9.7)$ & 0.03 \\
\hline Female vs male & $3.7(1.3-10.7)$ & 0.02 \\
\hline Stage 4 vs stages $0-3$ & $2.3(0.8-6.7)$ & 0.14 \\
\hline Control vs treatment & $2.6(0.9-7.8)$ & 0.09 \\
\hline Working vs not working & $0.4(0.1-1.1)$ & 0.08 \\
\hline$\leq \mathrm{HS}$ vs $>\mathrm{HS}$ & $1.3(0.3-4.9)$ & 0.72 \\
\hline Minority vs nonminority & I.3 (0.3-5.9) & 0.73 \\
\hline Age + I vs age & $0.9(0.9-1.0)$ & 0.02 \\
\hline Married/partnered vs not & $0.09(0.02-0.5)$ & 0.004 \\
\hline I-Month increase in time on therapy ${ }^{a}$ & I.2(0.8-I.5) & 0.29 \\
\hline
\end{tabular}

Notes: andicates a one-unit increase in the respective continuous scale; $\mathrm{N}=70$. Abbreviations: $\mathrm{Cl}$, confidence interval; $\mathrm{HS}$, high school; OR, odds ratio; $\mathrm{PHQ9C}$, Patient Health Questionnaire 9 continuous; SDS- I5, I5-item Symptom Distress Scale.

\section{Discussion}

In a sample of participants undergoing active cancer therapy, we found univariate evidence of associations with oral medication adherence, which included lack of a spouse/ partner, symptom distress, younger age, not working at the start of therapy, female sex, and oral chemotherapy vs oral hormonal medications. Our classification results suggest that significant relationships exist between adherence and symptom distress, age, working status at the start of therapy, sex, and relationship status.

Studies in oral agents for cancer treatment have documented findings similar to ours. Lebovits et al ${ }^{18}$ evaluated adherence to oral cytoxan and prednisone in women with breast cancer and found significant associations of psychological and physical symptoms with lower adherence. The influence of side effects on adherence have been documented with mixed results. Most oncology adherence studies have involved women with breast cancer on tamoxifen or aromatase inhibitors or patients with chronic myeloid leukemia on targeted oral agents. ${ }^{4}$ This limits a discussion of whether side effects affect oral medications in general. Virtually all studies, including ours, included concomitant measures of adherence and symptoms/side effects in which attribution of the side effect to the oral agent or another etiology is not clear. Nonetheless, high symptom burden is likely to affect selfadministration of oral medications, just as severe symptoms prompt emergency department visits and unscheduled admissions for patients with cancer seeking assistance. ${ }^{19}$ Hershman et $\mathrm{al}^{20}$ reported significantly earlier discontinuation of adjuvant hormonal therapy in unmarried (vs married) women with breast cancer. In contrast to our findings, patients with chronic myeloid leukemia in Belgium were evaluated during therapy with oral imatinib, and the study reported lower adherence in 


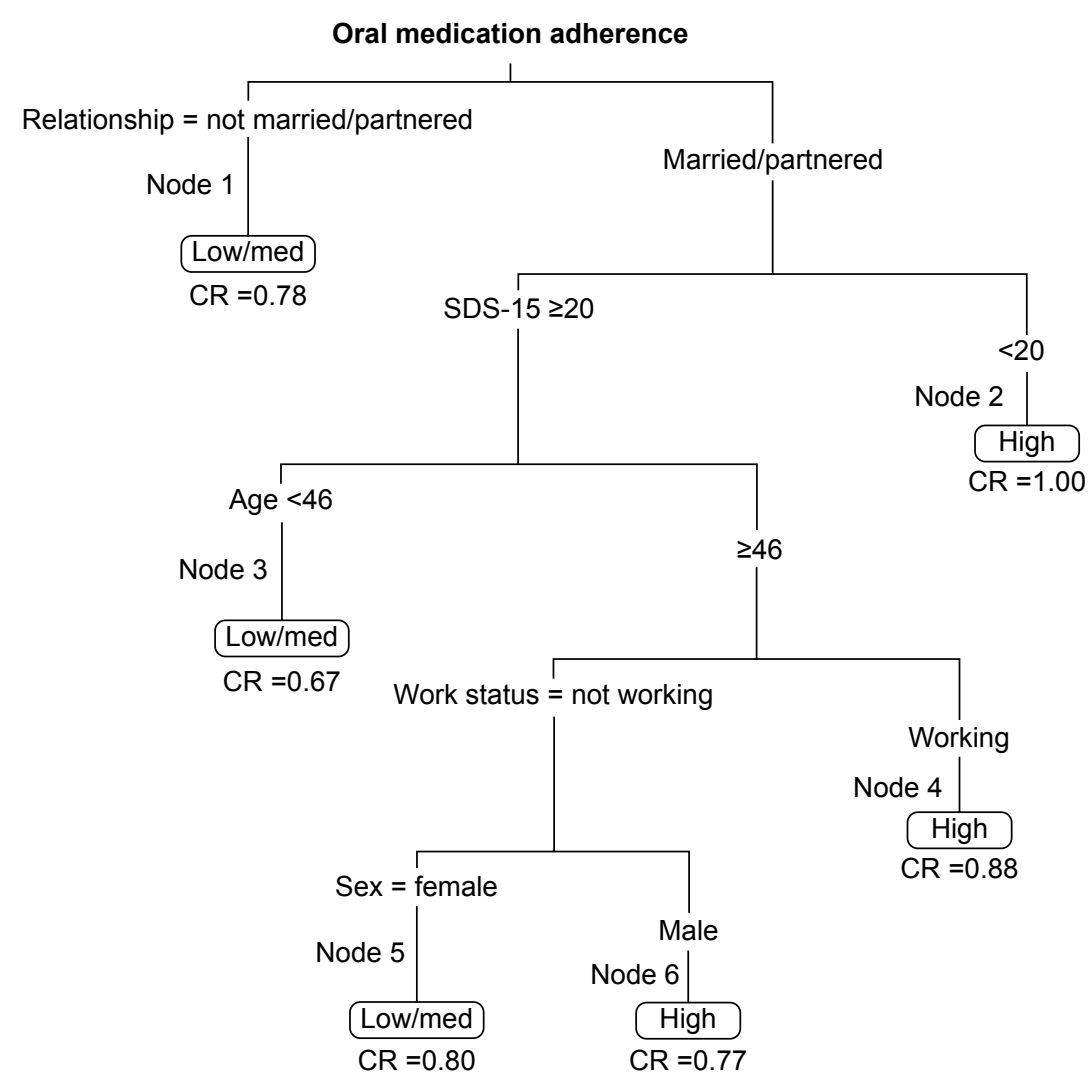

Figure I Classification tree: significant partitioning predictive of adherence.

Note: $\mathrm{N}=70$.

Abbreviations: CR, classification rate; med, medium; SDS-15, I5-item Symptom Distress Scale.

older patients and men; however, lower adherence was found also in those living without a spouse/partner. ${ }^{21}$

Working vs not working at the initiation of cancer therapy is a demographic variable we have found influential for several outcomes; ${ }^{10,19,22}$ yet we have not found reports from other investigators that documented the working status in relationship to oral agent adherence. The beneficial influence of working may be related to the structure of the workday, in which routine daily activities provide a framework into which medication self-administration fits well. Vance et $\mathrm{al}^{23}$ reviewed the literature on the benefits of employment for health outcomes in those with human immunodeficiency virus infection. The authors concluded that working promotes neurocognitive reserve and that effect may be manifested in several outcomes such as social engagement, regular sleep, less depression, and medication adherence. Future research in oncology could explore the protective effect of working in all patients with cancer, particularly in those at risk for cognitive impairment.

While the ESRA-C intervention did not directly prompt participants to adhere to self-administered oral medications, we saw some signal that the intervention was associated with higher adherence to oral medications. This was probably an indirect effect in that the ESRA-C intervention group experienced significantly lower symptom distress ${ }^{10}$ over the course of therapy, and lower depression ${ }^{24}$ at the end of therapy, than the control group, and both of these outcomes may have promoted adherence.

Our analysis and results are limited by the lack of racial and ethnic diversity in the sample. We did not record or include the complexity of dosing schedule, number of pills per day, or number of other medications taken, all of which may have had an influence on adherence.

When encountering patients who are unmarried or without a partner, clinicians may want to have more in-depth conversations about strategies for assuring adherence. The Multinational Association of Supportive Care in Cancer Oral Agent Teaching Tool ${ }^{25}$ has been demonstrated to be a useful and feasible tool in clinical cancer settings, ${ }^{26}$ as it covers the very type of issues that our findings suggest as contributors to nonadherence, notably cancer symptoms.

\section{Conclusion}

Nonadherence to therapeutic oral medications self-administered by patients with cancer was associated with being unmarried/ 
unpartnered. For those who were married/partnered, symptom distress, younger age, not working, and female sex all contributed to lower adherence. These findings may be used to identify patients at risk for nonadherence and subsequently provide additional support to these individuals.

\section{Acknowledgment}

This trial has been registered on Clinicaltrials.gov under registration identifier NCT00852852 (http://clinicaltrials. gov/ct2/show/NCT00852852; archived by WebCite at http:// www.webcitation.org/6YwAfwW17).

\section{Disclosure}

The authors report no conflicts of interest in this work.

\section{References}

1. Hershman DL, Shao T, Kushi LH, et al. Early discontinuation and nonadherence to adjuvant hormonal therapy are associated with increased mortality in women with breast cancer. Breast Cancer Res Treat. 2011;126(2):529-537.

2. Neuss MN, Polovich M, McNiff K, et al. 2013 updated American Society of Clinical Oncology/Oncology Nursing Society chemotherapy administration safety standards including standards for the safe administration and management of oral chemotherapy. Oncol Nurs Forum. 2013;40(3):225-233.

3. Levine AM, Richardson JL, Marks G, et al. Compliance with oral drug therapy in patients with hematologic malignancy. J Clin Oncol. 1987;5(9):1469-1476.

4. Johnson LA. Factors influencing oral adherence: qualitative metasummary and triangulation with quantitative evidence. Clin J Oncol Nurs. 2015;19(3):6-30.

5. Mathes T, Pieper D, Antoine SL, Eikermann M. Adherence influencing factors in patients taking oral anticancer agents: a systematic review. Cancer Epidemiol. 2014;38(3):214-226.

6. Bassan F, Peter F, Houbre B, et al. Adherence to oral antineoplastic agents by cancer patients: definition and literature review. Eur J Cancer Care. 2014;23(1):22-35.

7. Kavookjian J, Wittayanukorn S. Interventions for adherence with oral chemotherapy in hematological malignancies: a systematic review. Res Social Adm Pharm. 2015;11(3):303-314.

8. Mathes T, Antoine SL, Pieper D, Eikermann M. Adherence enhancing interventions for oral anticancer agents: a systematic review. Cancer Treat Rev. 2014;40(1):102-108.

9. Spoelstra SL, Sansoucie H. Putting evidence into practice: evidencebased interventions for oral agents for cancer. Clin J Oncol Nurs. 2015;19(3):60-72.

10. Berry DL, Hong F, Halpenny B, et al. Electronic self-report assessment for cancer and self-care support: results of a multicenter randomized trial. J Clin Oncol. 2014;32(3):199-205.
11. McCorkle R, Cooley M, Shea J. A User's Manual for the Symptom Distress Scale. New Haven: Yale University; 2000.

12. Kroenke K, Spitzer RL, Williams JB. The PHQ-9: validity of a brief depression severity measure. J Gen Intern Med. 2001;16(9):606-613.

13. Fann JR, Berry DL, Wolpin S, et al. Depression screening using the Patient Health Questionnaire-9 administered on a touch screen computer. Psychooncology. 2009;18(1):14-22.

14. Morisky DE, Ang A, Krousel-Wood M, Ward HJ. Predictive validity of a medication adherence measure in an outpatient setting. J Clin Hypertens. 2008;10(5):348-354.

15. Sommers RM, Miller K, Berry DL. Feasibility pilot on medication adherence and knowledge in ambulatory patients with gastrointestinal cancer. Oncol Nurs Forum. 2012;39(4):E373-E379.

16. James G, Witten D, Hastie T, Tibshiran R. An introduction to statistical learning: with applications in R. In: Casella G, Fienberg S, Olkin I, editors. Springer Texts in Statistics. New York: Springer Science+Business Media LLC; 2014. Available from: http://www-bcf. usc.edu/ gareth/ISL/ISLR\%20Fourth\%20Printing.pdf

17. Therneau TM, Atkinson EJ. An introduction to recursive partitioning using the RPART routines; 2015. Available from: http://cran.r-project. org/web/packages/rpart/vignettes/longintro.pdf. Accessed June 29, 2015.

18. Lebovits AH, Strain JJ, Schleifer SJ, Tanaka JS, Bhardwaj S, Messe MR. Patient noncompliance with self-administered chemotherapy. Cancer. 1990;65(1):17-22.

19. Siefert M, Blonquist T, Berry DL, Hong F. Symptom-related emergency department visits and hospital admissions during ambulatory cancer treatment. J Comm Supp Oncol. 2015;13:188-194.

20. Hershman DL, Kushi LH, Shao T, et al. Early discontinuation and nonadherence to adjuvant hormonal therapy in a cohort of 8,769 earlystage breast cancer patients. J Clin Oncol. 2010;28(27):4120-4128.

21. Noens L, van Lierde MA, De Bock R, et al. Prevalence, determinants, and outcomes of nonadherence to imatinib therapy in patients with chronic myeloid leukemia: the ADAGIO study. Blood. 2009;113(22): 5401-5411.

22. Artherholt SB, Hong F, Berry DL, Fann JR. Risk factors for depression in patients undergoing hematopoietic cell transplantation. Biol Blood Marrow Transplant. 2014;20(7):946-950.

23. Vance DE, Cody SL, Yoo-Jeong M, Jones GL, Nicholson WC. The role of employment on neurocognitive reserve in adults with HIV: a review of the literature. J Assoc Nurses AIDS Care. 2015;26(4):316-329.

24. Berry DL, Hong F, Halpenny B. Psychosocial outcomes of the electronic self-report assessment for cancer-II randomized trial. Paper presented at: American Society of Clinical Oncology Annual Meeting; June 1, 2015; Chicago.

25. Kav S, Schulmeister L, Nirenberg A, Barber L, Johnson J, Rittenberg C. Development of the MASCC teaching tool for patients receiving oral agents for cancer. Support Care Cancer. 2010;18(5): 583-590.

26. Boucher J, Lucca J, Hooper K, Pedulla L, Berry D. A Structured nursing intervention to address oral chemotherapy adherence in patients with non-small cell lung cancer. Oncol Nurs Forum. 2015;42(4): 383-389.
Patient Preference and Adherence

\section{Publish your work in this journal}

Patient Preference and Adherence is an international, peer-reviewed, open access journal that focuses on the growing importance of patient preference and adherence throughout the therapeutic continuum. Patient satisfaction, acceptability, quality of life, compliance, persistence and their role in developing new therapeutic modalities and compounds to optimize

\section{Dovepress}

clinical outcomes for existing disease states are major areas of interest for the journal. This journal has been accepted for indexing on PubMed Central. The manuscript management system is completely online and includes a very quick and fair peer-review system, which is all easy to use. Visit http://www. dovepress.com/testimonials.php to read real quotes from published authors. 